C ORR E S P O N D E N C E

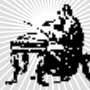

\title{
A Novel Familial Cardiac Arrhythmia Syndrome with Widespread ST-Segment Depression
}

TO THE EDITOR: Several classic cardiac genetic disorders have been identified from specific electrocardiographic (ECG) patterns. ${ }^{1-3}$ Here, we describe five unrelated families, from three different countries, with features that appear to represent a previously unrecognized autosomal dominant syndrome. These families were identified at three tertiary referral centers for patients with known or suspected inherited cardiac disorders (as described in the Supplementary Appendix, available with the full text of this letter at NEJM.org).

The index patient of Family A was followed regularly for more than 30 years after his incidental presentation at 36 years of age with an ECG showing deep and persistent, concave-upward ST-segment depression in leads I, II, aVL, aVF, and V2 through V6 (Fig. 1A). The ECG changes remained stable over time (Fig. S1A in the Supplementary Appendix) but worsened during exercise (Fig. S1B in the Supplementary Appendix). The patient remained asymptomatic until atrial fibrillation developed at 55 years of age. Eight

THIS WEEK'S LETTERS

1780 A Novel Familial Cardiac Arrhythmia Syndrome with Widespread ST-Segment Depression

1782 Lethal Fentanyl and Cocaine Intoxication

1783 Prehospital Plasma during Air Medical Transport in Trauma Patients

1784 Copeptin in the Diagnosis of Diabetes Insipidus

e33 Medicare's Bundled Payments Initiative for Medical Conditions

e34 A 15-Year-Old Girl with Acute Kidney Injury years later, he had aborted sudden cardiac death due to ventricular fibrillation. His son and daughter had similar ECG changes, as did four additional family members (Fig. 1B, and Fig. S1C and S1D in the Supplementary Appendix). Two relatives died from sudden cardiac death. Atrial fibrillation and ventricular arrhythmias were observed in the fifth decade of life in three family members. No coronary artery disease was identified in the affected persons, and imaging showed no, or only mild, left ventricular dysfunction.

The proband of Family B presented at 16 years of age with syncopal episodes and similar STsegment changes (Fig. S2 in the Supplementary Appendix). Incessant ventricular tachycardia (including torsades des pointes) developed, then ventricular-fibrillation arrests and moderate left ventricular dysfunction. He died at 17 years of age after the failure of a heart transplantation performed for intractable arrhythmia. His father and uncle, with nearly identical ECG changes, were diagnosed with atrial fibrillation in their early 60s; the father later received an implantable cardioverter-defibrillator for episodes of ventricular tachycardia or ventricular fibrillation. Four younger, asymptomatic relatives (the youngest was 6 years of age) have extremely similar ECG changes. Three other families, with a total of nine affected persons, were identified with very similar ECG changes and similar arrhythmias (Figs. S3 through S5 in the Supplementary Appendix). Details of the clinical characteristics of the members of all five families are provided in the Supplementary Appendix, including in Tables S1 and S2.

Genetic evaluations were performed at the discretion of the referring physician. Gene panels that included genes associated with cardiovas- 
cular disease were screened in all five families (Table S3 in the Supplementary Appendix); all genetic tests were negative.

In conclusion, we identified five families with an autosomal dominant cardiac syndrome characterized by rather uniform ECG changes with marked, persistent, nonischemic ST-segment depression, the development of atrial fibrillation and ventricular arrhythmias, and (in older persons) some degree of left ventricular dysfunction. In contrast to some other genetic disorders (e.g., the long-QT syndrome and the Brugada syndrome) that are characterized by dynamic pathognomonic ECG changes, ${ }^{4,5}$ the ST-segment depression observed here remained stable over time. On the basis of the findings in these five families, we propose diagnostic criteria for this novel syndrome (Table S4 in the Supplementary Appendix).

Henning Bundgaard, M.D., D.M.Sc.

Christian Jøns, M.D., Ph.D.

Copenhagen University Hospital

Copenhagen, Denmark

henning.bundgaard@regionh.dk

Elisabeth M. Lodder, Ph.D.

Amsterdam University Medical Center

Amsterdam, the Netherlands

Jose M.G. Izarzugaza, Ph.D.

Jose A. Romero Herrera, M.Sc.

Technical University of Denmark

Copenhagen, Denmark

Steen Pehrson, M.D., D.M.Sc.

Jacob Tfelt-Hansen, M.D., D.M.Sc.

Copenhagen University Hospital

Copenhagen, Denmark

Gustav Ahlberg, M.Sc.

Morten S. Olesen, Ph.D.

Anders G. Holst, M.D., Ph.D.

University of Copenhagen

Copenhagen, Denmark

Hein Wellens, M.D., Ph.D.

Cardiovascular Research Center

Maastricht, the Netherlands

Carin de Villiers, Ph.D.

Robert Hastings, M.B., Ch.B., D.Phil.

University of Oxford

Oxford, United Kingdom

Graham Stuart, M.B., Ch.B.

Bristol Heart Institute

Bristol, United Kingdom

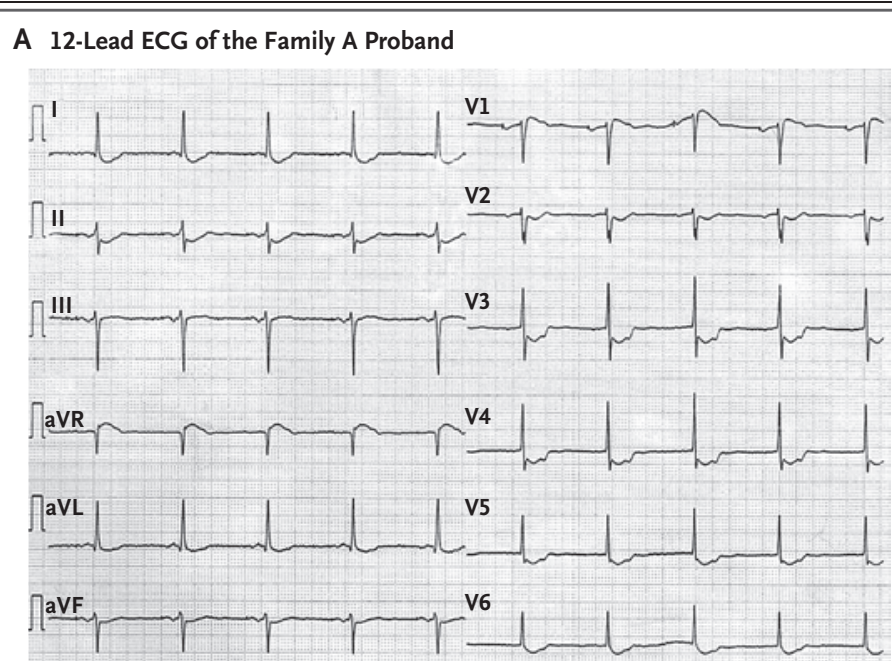

B Pedigree of Family A

I

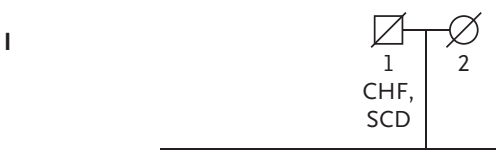

II

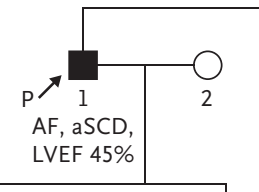

III
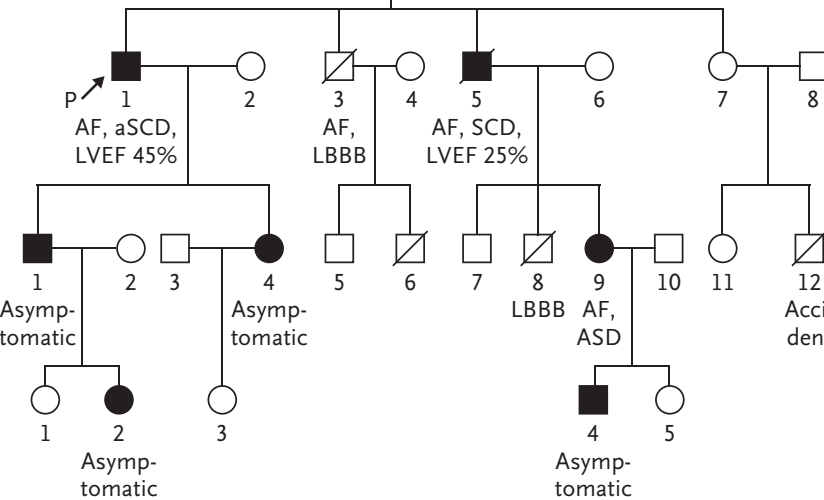

Figure 1. ECG Characteristics and Pedigree in the First Family Identified with the Familial ST-Segment Depression Syndrome.

In Family A, the proband's 12-lead ECG (Panel A), recorded at 63 years of age, shows atrial paced rhythm; concave-upward ST-segment depression in leads I, II, aVL, aVF, and V2 through V6; and ST-segment elevation in lead aVR. A notch was observed in the ascending part of the ST segment. Also shown is the pedigree of Family A (Panel B). Circles indicate female persons, squares male persons, solid symbols affected persons, open symbols unaffected persons, and slashes deceased persons; the proband $(P)$ is noted with an arrow. AF denotes atrial fibrillation, aSCD aborted sudden cardiac death, ASD atrial septal defect, CHF congestive heart failure, LBBB left bundle-branch block, LVEF left ventricular ejection fraction, and SCD sudden cardiac death. 
Søren Brunak, Ph.D.

University of Copenhagen

Copenhagen, Denmark

Arthur A.M. Wilde, M.D., Ph.D.

Amsterdam University Medical Center

Amsterdam, the Netherlands

Hugh Watkins, M.D., Ph.D.

University of Oxford

Oxford, United Kingdom

Alex H. Christensen, M.D., Ph.D.

Herlev-Gentofte Hospital

Copenhagen, Denmark

Drs. Wilde, Watkins, and Christensen contributed equally to this letter.

Supported by grants from the AP Moller Foundation and the Research Foundation at Rigshospitalet (to Dr. Bundgaard); the Novo Nordisk Foundation (NNF17OC0027594 and NNF14CC0001) and the Innovation Fund Denmark (5184-00102B) (to Dr. Izarzugaza, Mr. Romero Herrera, and Dr. Brunak); the Netherlands CardioVascular Research Initiative, the Dutch Heart Foundation, the Dutch Federation of University Medical Centers, the Netherlands Organization for Health Research and Development, and the Royal Netherlands Academy of Sciences (to Drs. Lodder and Wilde); and the National Institute for Health Research Oxford Biomedical Research Centre (to Dr. Watkins).

Disclosure forms provided by the authors are available with the full text of this letter at NEJM.org.

1. Jervell A, Lange-Nielsen F. Congenital deaf-mutism, functional heart disease with prolongation of the Q-T interval and sudden death. Am Heart J 1957;54:59-68.

2. Brugada P, Brugada J. Right bundle branch block, persistent ST segment elevation and sudden cardiac death: a distinct clinical and electrocardiographic syndrome - a multicenter report. J Am Coll Cardiol 1992;20:1391-6.

3. Haïssaguerre M, Derval N, Sacher F, et al. Sudden cardiac arrest associated with early repolarization. N Engl J Med 2008; 358:2016-23.

4. Nemec J, Buncová M, Shusterman V, Winter B, Shen W-K, Ackerman MJ. QT interval variability and adaptation to heart rate changes in patients with long QT syndrome. Pacing Clin Electrophysiol 2009;32:72-81.

5. Richter S, Sarkozy A, Veltmann C, et al. Variability of the diagnostic ECG pattern in an ICD patient population with Brugada syndrome. J Cardiovasc Electrophysiol 2009;20:69-75.

DOI: 10.1056/NEJMc1807668 